\title{
Homotypic Regulation of Neuronal Morphology and Connectivity in the Mouse Retina
}

\author{
Sammy C. S. Lee, ${ }^{1}$ Erin J. Cowgilll, ${ }^{1,2}$ Ali Al-Nabulsi, ${ }^{1,2}$ Emma J. Quinn, ${ }^{1,2}$ Sylvia M. Evans, ${ }^{3}$ and Benjamin E. Reese ${ }^{1,2}$ \\ ${ }^{1}$ Neuroscience Research Institute and ${ }^{2}$ Department of Psychological \& Brain Sciences, University of California, Santa Barbara, California 93106, and \\ ${ }^{3}$ Department of Pharmacology, School of Pharmacy and Pharmaceutical Sciences, University of California, San Diego, California 92093
}

The establishment of neuronal circuitry during development relies upon the action of cell-intrinsic mechanisms that specify neuronal form as well as plastic processes that require the transmission of neural activity between afferents and their targets. Here, we examine the role of interactions between neighboring like-type cells within the mouse retina upon neuronal differentiation and circuit formation. Two different genetically modified mouse models were used to modulate the density of homotypic neighbors, the Type 7 cone bipolar cells, without affecting the density of their afferents, the cone photoreceptors. We demonstrate a corresponding plasticity in dendritic field area when the density of Type 7 cone bipolar cells is elevated or reduced. In accord with this variation in dendritic field area across an invariant population of afferents, individual Type 7 cone bipolar cells are also shown to modulate the number of cone pedicles contacted without varying the number of contacts at each cone pedicle. Analysis of developing Type 7 cone bipolar cells reveals that the dendritic tiling present in maturity is achieved secondarily, after an initial stage of dendritic overlap, when the dendritic terminals are stratified at the level of the cone pedicles but are not localized to them. These results demonstrate a conspicuous developmental plasticity in neural circuit formation independent of neural activity, requiring homotypic interactions between neighboring cells that ultimately regulate connectivity within the retina.

\section{Introduction}

The complex processing of the nervous system depends upon an intricate connectivity established during early development. Multiple processes coordinate the assembly of this precise circuitry, including cell-intrinsic instructions that direct and constrain morphogenesis (Jan and Jan, 2010), and activity-dependent mechanisms that establish the synaptic contacts underlying these neural circuits (Holtmaat and Svoboda, 2009). Neural transmission has been shown to play a substantial role in the refinement of synaptic connectivity between afferent neurons and their targets during development, most notably within the primary visual cortex and the cerebellum and at the neuromuscular junction (Bleckert and Wong, 2011). Such activity-dependent processes have been shown to regulate the differentiation of axonal arbors, the patterning of dendritic processes, and the distribution and density of synaptic contacts (Espinosa et al., 2009; Kerschensteiner et al., 2009; Li et al., 2011). Here, by contrast, we show a degree of dendritic plasticity that is independent of afferent activity, relying instead upon the local presence of like-type neighbors. This latter interaction is demon-

\footnotetext{
Received June 7, 2011; revised July 21, 2011; accepted Aug. 15, 2011.

Author contributions: S.C.S.L. and B.E.R. designed research; S.C.S.L. performed research; S.M.E. contributed unpublished reagents/analytic tools; S.C.S.L., E.J.C., A.A.-N., E.J.Q., and B.E.R. analyzed data; S.C.S.L. and B.E.R. wrote the paper.

This work was supported by the NIH (EY-19968 and RR-22585) and by a National Health \& Medical Research Council (Australia) Overseas Biomedical Fellowship (567031) to S.C.S.L.

Correspondence should be addressed to Benjamin E. Reese, Neuroscience Research Institute, University of California, Santa Barbara, CA 93106-5060. E-mail: breese@psych.ucsb.edu.

DOI:10.1523/JNEUROSCI.2844-11.2011

Copyright $\odot 2011$ the authors $\quad 0270-6474 / 11 / 3114126-08 \$ 15.00 / 0$
}

strated to be a major determinant of the connectivity between target neurons and their afferents at the first synapse in the visual pathway, that between the cone photoreceptors and their target neurons, the cone bipolar cells.

Cone bipolar cells in the retina receive convergent innervation from a local population of cone photoreceptors via a dendritic arbor that selectively targets the pedicles of these cones (Haverkamp et al., 2000). There are multiple types of cone bipolar cell at every location on the retina, with each type producing a dendritic arbor of a particular size and having a characteristic connectivity associated with the types of cones innervated by it (Breuninger et al., 2011). Cone bipolar cells of the same type colonize neighboring territories within the outer plexiform layer (OPL), though occasionally sharing contacts with individual pedicles lying at the boundary of each homotypic dendritic field (Wässle et al., 2009). At each pedicle, multiple cone bipolar cell types establish dendritic contacts that serve as the postsynaptic sites for neural transmission.

The present study has asked whether the size of the dendritic arbor of a cone bipolar cell is commanded by the local density of like-type neighboring cells. Dendritic fields that tile a structure are commonly assumed to regulate their dendritic growth through some form of homotypic recognition, yet direct tests of this expectation have suggested cell-intrinsic regulation of dendritic field size (Lin et al., 2004). Consequently, we examined the effects of either decreasing or increasing local homotypic density upon dendritic differentiation for a particular type of cone bipolar cell in the mouse retina. In addition, we have examined the consequences of such dendritic plasticity upon the connectivity of these cells: might cell-intrinsic constraints limit the number of 

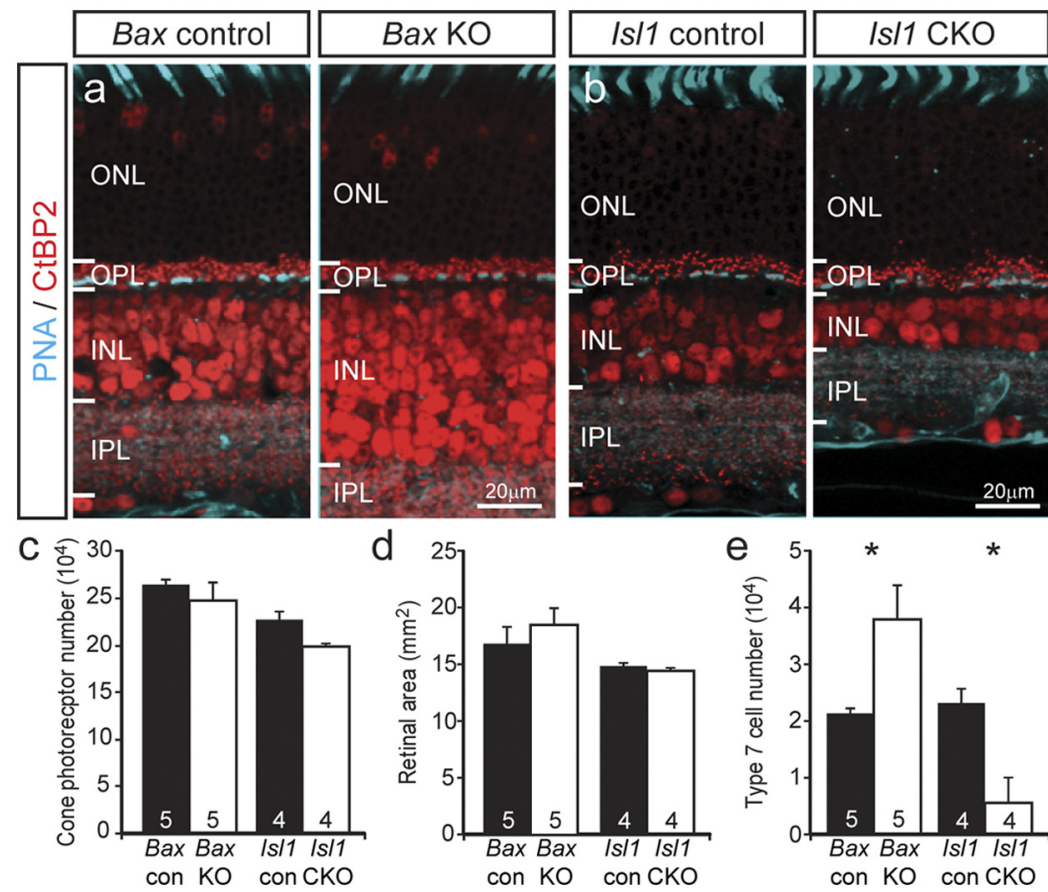

Figure 1. Bax and $/ s / 1$ mutant retinas modulate Type 7 cone bipolar density without affecting the density of afferent innervation. $\boldsymbol{a}, \boldsymbol{b}$, Transverse sections from Bax control and Bax KO retinas and from $/ s / 7$ control and $/ s / 1$ CKO retinas stained with antibodies against CtBP2 (red) and PNA (cyan). There is no change in thickness of the outer retinal layers (ONL and OPL) for both conditions when compared to their respective controls. There is, however, an increase in thickness in the inner layers (INL and IPL) of the Bax $K 0$ retina compared to its littermate control, and a decrease in the $/ s / 1$ CKO relative to its control. $\boldsymbol{c}, \boldsymbol{d}$, To confirm that there is no change in the number of cone photoreceptors in the mutant retinas, their total numbers were estimated in retinal whole mounts and compared with respective control retinas. Neither total cone photoreceptor number nor retinal area was significantly different. $\boldsymbol{e}$, These mice were also crossed with GUS8.4GFP mice, from which the total number of Type 7 cells (that were GFP-positive) was estimated in the same whole mounts. Bax KO retinas showed nearly a $60 \%$ increase in the total number of Type 7 cells when compared to controls $(p<0.05)$, while $/ s / 1$ CKO retinas showed a $>75 \%$ reduction $(p<0.01)$. Means and SEs are indicated, and the number of retinas sampled (each from a different mouse) is indicated for each group.

afferents contacted or the number of dendritic terminals produced by single cone bipolar cells? Finally, we have examined the dendritic organization and connectivity of cone bipolar cells during development, including pairs of adjacent neighbors, to determine the relationship between homotypic regulation of dendritic field size and afferent innervation as synaptic connectivity begins to materialize.

\section{Materials and Methods}

Animals. Mice carrying the gene for green fluorescent protein (GFP) driven by an $8.4 \mathrm{~kb}$ promoter region upstream from the $\alpha$-gustducin gene (GUS8.4GFP mice) were used for targeting Type 7 cone bipolar cells in the retina (Huang et al., 2003). To study the effects of homotypic density upon cone bipolar dendritic field size, we crossed GUS8.4GFP mice with two genetically modified mice, $\mathrm{B} 6.129 \times 1-\mathrm{Bax}^{\mathrm{tm} 1 \mathrm{Sjk} / \mathrm{J}}$ (hereafter Bax KO) mice and Islet-1 (hereafter Isl1) conditional knock-out (CKO) mice, that show changes in the number of inner retinal neurons without changing the number of outer retinal neurons. The resulting F2 generations of these crosses were identified by PCR and littermates were used as controls. Homozygous GUS8.4GFP animals were also used to compare developing (P13) versus mature (P30) Type 7 cone bipolar cells in wild-type retinas. To determine whether visual function participates in cone bipolar dendritic differentiation, we also crossed the GUS8.4GFP mice with mice from the ALS/LtJ strain, containing a mutation in Gnat2 [guanine nucleotide-binding protein (G-protein)], the gene encoding for the $\alpha$-subunit of transducin (Chang et al., 2006). Bax KO and ALS/LtJ mice were obtained from The Jackson Laboratory, while the GUS8.4GFP mice were kindly provided by Dr. Richard Masland (Harvard Medical School, Cambridge, MA). A retinaspecific Cre-line, Chx10-GFPCre ${ }^{\text {tg } /+}$ mice (Rowan and Cepko, 2004), was also obtained from The Jackson Laboratory and was crossed with Isll floxfflox mice imported from University of California San Diego (Sun et al., 2007) to generate Chx10-GFPCre ${ }^{\text {tg/ }}$; Isl ${ }^{f l o x /+}$ mice. These subsequent mice were mated with each other, and their litters were genotyped using PCR to generate Chx10-

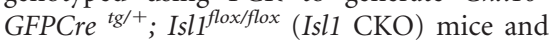
littermate controls. Mice of either sex were used, in all experiments.

Tissue preparation. All experiments were conducted under authorization of the Institutional Animal Care and Use Committee at University of California Santa Barbara and in accord with the NIH Guide for the Care and Use of Laboratory Animals. Mice were killed with sodium pentobarbital $(120 \mathrm{mg} / \mathrm{kg}$, i.p.), and the eyes were enucleated and immersion fixed in $4 \%$ paraformaldehyde in $0.1 \mathrm{~m}$ sodium phosphate buffer $\left(\mathrm{pH} 7.2\right.$ at $20^{\circ} \mathrm{C}$ ) for $30 \mathrm{~min}$. The eyecups were then washed in $0.1 \mathrm{M}$ sodium phosphate buffer $(\mathrm{PB})$ and retinas dissected as whole mounts for injections or prepared for vibratome sectioning. For single-cell injections, whole retinas were transferred to a fixed-stage Nikon Eclipse E600 epifluorescence microscope and the axons of GFP-positive Type 7 cells were impaled with a micropipette filled with $0.5 \%$ DiI $\left(1,1^{\prime}\right.$,dioctadecyl-3,3,3',3'-tetramethylindocarbocyanine perchlorate) dissolved in 100\% ethanol as previously described (Keeley and Reese, 2010). Retinas were subsequently labeled with peanut agglutinin (PNA) conjugated with Alexa Fluor 647 in PBS overnight to label the active sites of cone pedicles.

Immunofluorescence. Immunolabeling was performed on retinal sections by flatmounting the retina on filter paper (Millipore), embedding in $5 \%$ agarose, and then sectioning on a Vibratome at $100 \mu \mathrm{m}$. The sections were incubated in a solution containing mouse monoclonal antibodies to C-terminal binding protein 2 (CtBP2; 1:10,000, BD Transduction), rabbit anti-GFP conjugated with Alexa Fluor 488 (1:1000, Invitrogen), and PNA conjugated with Alexa Fluor 647 (1:1000, Invitrogen) over two nights. Sections were then rinsed in PBS and incubated overnight with donkey anti-mouse IgG conjugated to Alexa 594 secondary antibodies (1:250, Invitrogen).

To quantify cell numbers, whole mounts were incubated over three nights in a solution containing PNA conjugated with Alexa Fluor 647 (1:1000; Invitrogen), goat polyclonal antibodies to GFP conjugated with FITC (1:250; Abcam), and rabbit polyclonal antibodies to PKC $\alpha$ (1: 10,000; Abcam) diluted in PBS. Retinas were then rinsed in PBS and further incubated with donkey anti-rabbit $\operatorname{IgG}$ conjugated to Alexa Fluor 594 secondary antibodies overnight. Retinas were rinsed in PBS and were then mounted flat on slides using Fluoro-Gel (Electron Microscopy Sciences). All incubation solutions contained 1\% Triton X-100 (exceptions to preparations with DiI), and these steps were performed at room temperature.

Imaging. Retinal fields used to quantify cell numbers and dendritic fields of DiI-labeled Type 7 cells (confirmed by retained GFP signal in their somas) were imaged using an Olympus Fluoview 1000 laser scanning confocal microscope with a $25 \times(1.4 \mathrm{NA})$ water-immersion objective at $0.5 \mu \mathrm{m}$ intervals through the $z$-axis. Captured image stacks were processed using AutoQuant (Media Cybernetics) and analyzed using MetaMorph (MDS Analytical Technologies) software. Retinal areas were measured by tracing the outlines of each retina using an Olympus BH2 microscope with an attached Sony video camera to a computer running BioQuant Nova Prime software (R\&M Biometrics).

Quantification. To quantify numbers of cone pedicles and Type 7 cells in Bax KO and Isll CKO mice and in their respective littermate controls, 

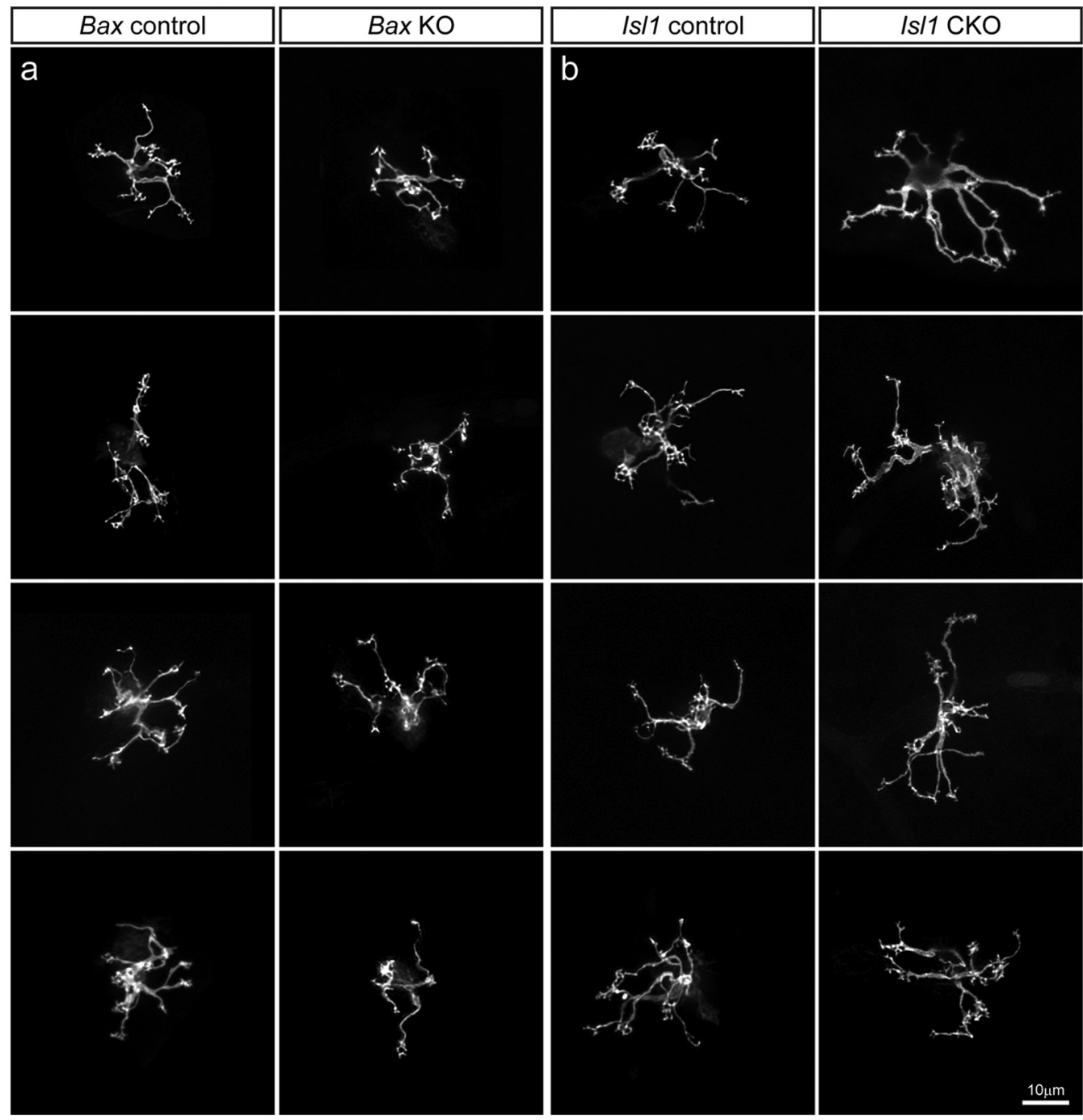

Figure 2. Type 7 cone bipolar cells establish a normal dendritic morphology despite variation in homotypic density. Examples of Type 7 cone bipolar cells in the Bax KO (a) and /s/ 7 CKO (b) retinas, and their respective control retinas, show them to be normal healthy cone bipolar cells, distinguished only by the fact that they are now smaller, or larger, in dendritic field area, respectively.

retinas were stained for PNA, anti-GFP, and PKC $\alpha$. PNA was used to identify cone pedicles while GFP-positive bipolar axons that were PKC $\alpha$ negative (confirming they are not rod bipolar axons) were used to identify Type 7 axons. The Type 7 cone bipolar cells were unambiguously discriminated from the GFP labeling associated with the Chx10-GFPCre reporter in the Isl1 CKO and littermate control mice because only the former GFP signal includes the dendritic and axonal arbor. Twelve fields, each of $16,139 \mu \mathrm{m}^{2}$ in size, were sampled (using MetaMorph software) at $0.5 \mathrm{~mm}, 1.0 \mathrm{~mm}$, and $1.5 \mathrm{~mm}$ from the optic nerve head in each quadrant of the whole-mounted retina. Counts from these regions were then converted into densities (cells per square millimeter), and the average density was then multiplied by retinal area to give an estimate of total number of cells for a given type.

For each DiI-injected Type 7 cell, the dendritic field size was measured by calculating the area of a convex polygon encompassing the dendritic arbor. In addition, by carefully reconstructing the dendritic morphology of each cell through the $z$-stack of images, the number of dendritic endings in the plane of the pedicles and the number of PNA-labeled cone pedicles contacted by each cell was determined. Dendritic terminals not contacting cone pedicles were also quantified. A Student's $t$ test was used to determine significance at the 0.05 level.

\section{Results}

To examine the role of homotypic interactions upon the differentiation of cone bipolar cell morphology, we labeled single cells of a particular type of cone bipolar cell, the Type 7 cell, in two different mouse models that modulate neuronal density within the inner retina without affecting the outer retina (Fig. 1a,b). Bax $\mathrm{KO}$ mice have increased neuronal densities in the retinal ganglion cell layer and inner nuclear layer (GCL and INL, respectively), due to the loss of this pro-apoptotic protein (Ogilvie et al., 1998; Péquignot et al., 2003), but show no change in the thickness of the outer nuclear layer (ONL), nor in the total number of cone photoreceptors therein (Fig. 1c). Isl1 CKO mice, on the other hand, have reduced neuronal densities in the GCL and INL due to the failure of a large percentage of these neuronal types to differentiate normally in the absence of this transcription factor (Elshatory et al., 2007b), but they still differentiate a normal ONL and total cone photoreceptor number (Fig. 1c). In the Isl1 CKO mice, the incomplete loss of a specific type of neuron is a consequence of the mosaic expression of cre-recombinase, leaving some variable proportion remaining (Elshatory et al., 2007b). Neither of these genetic manipulations compromises ocular growth, evidenced by the fact that retinal area is not significantly affected in either case (Fig. 1d).

To identify Type 7 cells selectively, both mouse models were crossed with a transgenic mouse carrying the gene for GFP down- 


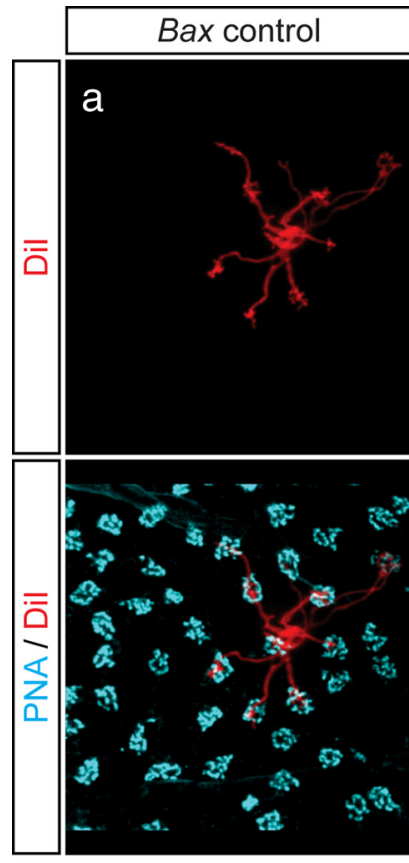

C

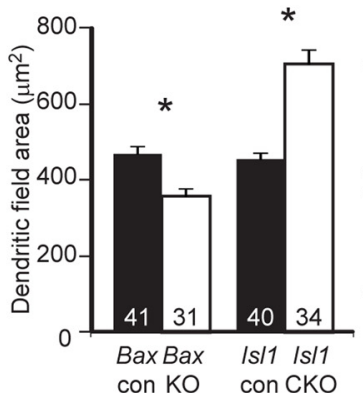

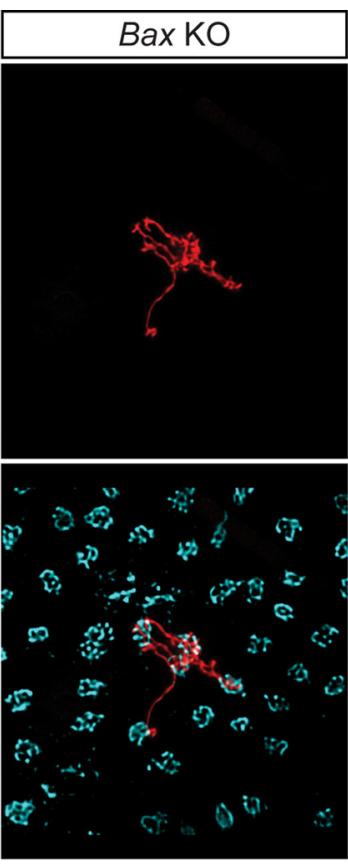

d

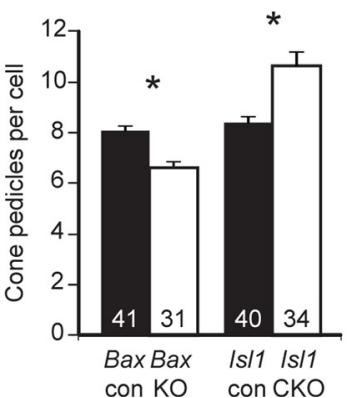

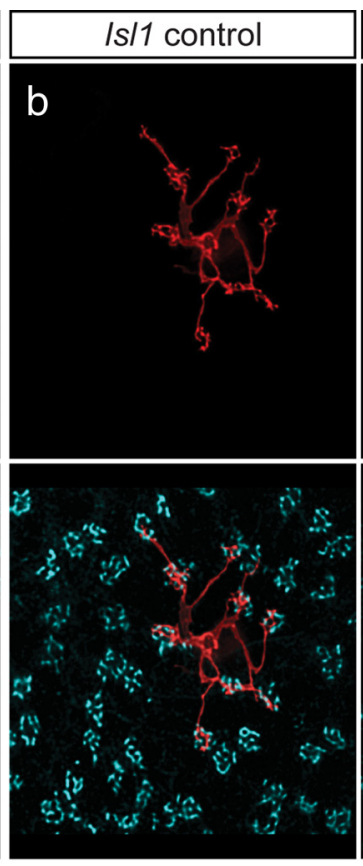

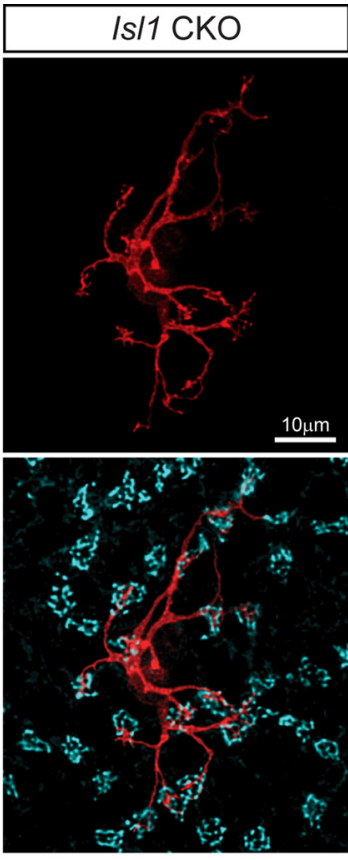

e

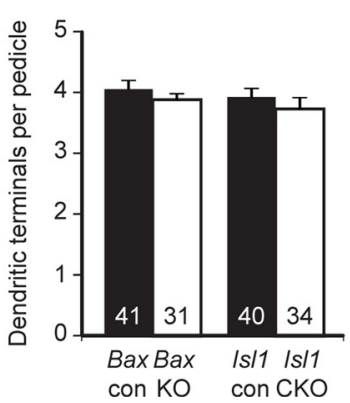

f

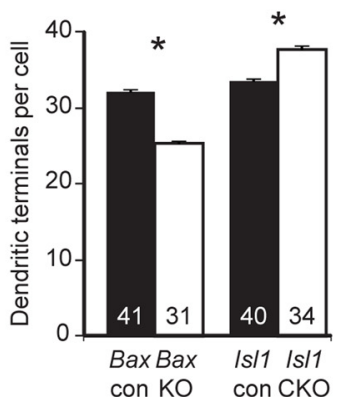

Figure 3. Dendritic field size and sampling of afferents are dependent on homotypic neighbors. $\boldsymbol{a}, \boldsymbol{b}$, Dendritic fields of Dil-labeled Type 7 cells (red) from Bax (a) and $/ s / 1$ (b) mutant retinas show a conspicuous difference in area when compared to their respective controls. $\boldsymbol{c}, \boldsymbol{d}$, Increasing the density of homotypic neighbors (in the Bax K0 retina) decreases dendritic field area $(p<0.001)$ and the number of cone pedicles (cyan) contacted $(p<0.001$ ), while decreasing homotypic neighbors (in the $/ s / 1$ CKO retina) leads to an increase in dendritic field area ( $p<0.0000001$ ) as well as the number of cone pedicles contacted $(p<0.001)$. $\boldsymbol{e}$, The synaptic relationship at each cone pedicle is, however, unchanged. $\boldsymbol{f}$, The total number of dendritic endings per cell must therefore be determined by homotypic density as well ( $p<0.001$ and $<0.05$, respectively). Means and SEs are indicated, as are the number of cells analyzed in each group.

stream of the $\alpha$-gustducin gene promoter (GUS8.4GFP mice) driving expression in this one type of cone bipolar cell and within rod bipolar cells. Each of these two types of labeled bipolar cell is readily discriminated by the intensity with which it expresses GFP, and by the dendritic arbor and axon terminal these two bipolar cells exhibit, the latter stratifying at different depths within the inner synaptic layer (Huang et al., 2003). We first confirmed that the density of Type 7 cells was indeed modulated in these two models, since not every inner retinal cell type is increased in the Bax KO retina (Whitney et al., 2008), nor are they all decreased in the Isl1 CKO retina (Whitney et al., 2011a). By counting the number of GFP-positive Type 7 cells in the same retinas for which cone photoreceptor numbers were determined, we confirmed a significant increase in the Bax KO retina and a significant decrease in the Isl1 CKO retina (Fig. 1e).

The distinctive axon terminals of the Type 7 cells can be selectively targeted for labeling with the lipophilic tracer, DiI, in fixed retinal whole mounts, yielding exquisite filling of the entire neuronal membrane, including the individual dendritic terminations invaginating into single pedicles (Keeley and Reese, 2010). Using this approach, we labeled single Type 7 cells in Bax KO and Isl1 CKO retinas, comparing them directly with those labeled in respective littermate control retinas (Fig. 2). Type 7 cells in the thicker Bax KO retina had significantly smaller dendritic arbors (Fig. 3c), though without appearing atrophic (Fig. 2a). These cells now contacted significantly fewer cone pedicles (Fig. $3 a, d$ ), but conserved their average number of terminal endings at each pedicle contacted (Fig. 3e), consequently producing a smaller total number of terminal endings across the entire dendritic arbor (Fig. 3f). The dendritic arbors of Type 7 cells in the Isl1 CKO retina, in comparison, were significantly larger (Fig. $3 c$ ) in these substantially thinner retinas, contacting a larger than normal number of cone pedicles (Fig. $3 b, d$ ). These cells also presented an otherwise normal, healthy appearance (Fig. $2 b$ ), despite the fact that the pedicles themselves were occasionally atrophic, presumably due to the overall reduction in other postsynaptic targets of the pedicles present in these thinner retinas (Elshatory et al., 2007b). The average number of contacts the remaining Type 7 cone bipolar cells made at each pedicle was not altered (Fig. 3e), yielding a larger than normal total number of postsynaptic contacts per Type 7 cell (Fig. $3 f$ ).

Type 7 cells, therefore, modulate their dendritic field areas in relation to homotypic cell density, and as a consequence, form contacts with pedicles falling within these modified dendritic ter- 


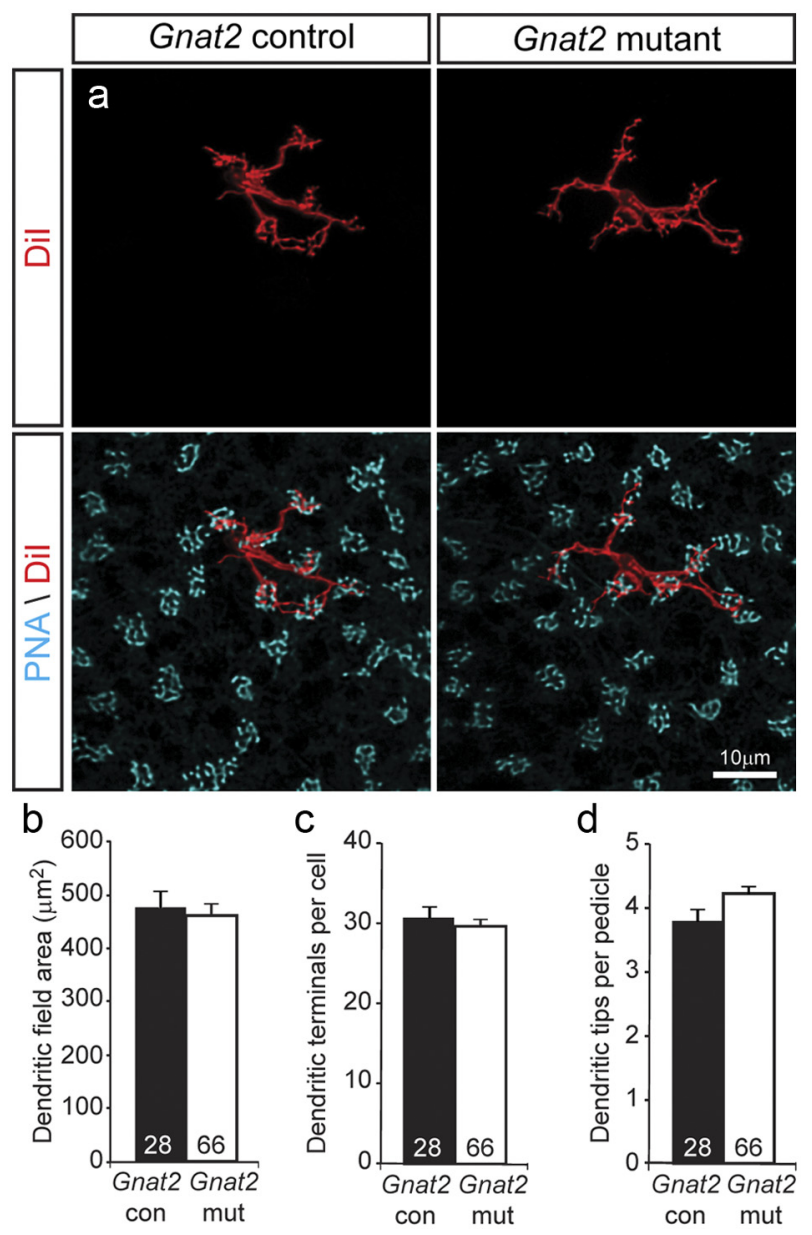

Figure 4. The establishment of dendritic morphology and connectivity of Type 7 cone bipolar cells does not require cone photoresponses. $\boldsymbol{a}$, Gnat2-mutant retinas have defective cone $\alpha$-transducin and thus are unresponsive to light. Dil-labeled Type 7 cells from Gnat2-mutant retinas are indiscriminable from those in littermate control retinas. $\boldsymbol{b}$ - $\boldsymbol{d}$, Dendritic field size, the number of dendritic terminals for each cell, and the average number of terminals per pedicle were all unaffected. Conventions are as in Figure 1.

ritories. The fact that the number of cones contacted and the number of total terminal endings made by a Type 7 cell varied in both conditions demonstrates these features to be established by the local environment, not by cell-intrinsic specification. They are not, however, dependent upon neural activity, but rather are the indirect consequences of homotypic regulation of dendritic field size. Since cone bipolar cells are known to differentiate late during postnatal development, in the second postnatal week (Morgan et al., 2006), when outer segments form and photoreceptors become functional (Olney, 1968; Sherry et al., 2003), we independently assessed any role of cone-mediated visual activity in the Gnat2-mutant mouse retina. These mice are deficient for cone $\alpha$-transducin and thus exhibit no cone photoresponse (Chang et al., 2006). Dendritic field size and terminal clustering at the pedicles were observed to differentiate normally in the Gnat2-mutant retina (Fig. 4a), confirming they do not require visually mediated activity driven by the cones for their formation nor for their maintenance (Fig. $4 b-d$ ).

One simple interpretation of these results is that the dendritic fields of neighboring Type 7 cells target cone pedicles as they differentiate, exhibiting contact inhibition with homotypic neighbors to ensure mutually exclusive territories from the outset. In fact, early differentiating Type 7 cells have larger than normal dendritic fields at P13 (Fig. 5a,b), contacting a larger number of pedicles than at later stages (Fig. 5c), though establishing a normal number of contacts per pedicle (Fig. $5 d, e$ ). Their dendritic arbors are, however, excessively branched (Fig. 6, arrowheads), extending significantly more dendritic endings that do not colocalize with any cone pedicles (compare bottom panels in Fig. $5 a$; Fig. $5 f$ ). As development proceeds, three changes take place: the presence of these interpedicle endings declines (Fig. $5 f$ ), while dendritic field size retracts (Fig. 5b), leaving fewer pedicles contacted (Fig. $5 c$ ). The implication of these latter two changes is that neighboring dendritic fields may initially overlap one another. The labeling of neighboring Type 7 cells shows this directly: adjacent Type 7 cells show a greater propensity to share contacts at neighboring pedicles and to encroach into neighboring dendritic terrains (Fig. $5 g$ ), relative to the discrete and exclusive colonization of individual pedicles that are more typical in the adult retina (Fig. 5h).

\section{Discussion}

The present study has shown conclusively that Type 7 cone bipolar cells regulate the size of their dendritic fields by interactions with their homotypic neighbors. Furthermore, it has demonstrated that the number of cone pedicles contacted by Type 7 cone bipolar cells is likewise commanded by dendritic field size, rather than being cell-intrinsically determined. It has also shown that the number of dendritic terminations per pedicle is invariant across conditions and through development. Finally, this study has revealed that the dendritic tiling of the Type 7 cone bipolar cells is not produced by a contact inhibition preventing dendritic overlap, but emerges following a period of dendritic overlap, before Type 7 cone bipolar dendrites selectively target the cone pedicles. Each of these new findings is explored in further detail below.

\section{Homotypic regulation of dendritic field size}

Cone bipolar cells of a particular type are thought to provide a uniform coverage of the retina by establishing a tiling of their dendritic fields: field areas extend up to, but do not overlap with, those from other homotypic neighbors, so that the population provides a coverage of 1.0 at every location upon the retina (Wässle et al., 2009). Of course, the presence of a dendritic coverage on the order of 1.0 is not, in itself, a demonstration that dendritic fields are sensitive to the presence of their homotypic neighbors, as has been shown directly for two population of retinal ganglion cells (Lin et al., 2004). There, genetic depletion of melanopsin-positive ganglion cells or of the SMI-32-positive ganglion cells by $80-95 \%$ does not alter the dendritic field size or patterning of remaining homotypic neighbors. The inverse assumption, that overlapping dendritic fields for a cell type must preclude such homotypic regulation, has also been shown to be incorrect: horizontal cells in the mouse retina display a dendritic coverage factor of 6.0 (Reese et al., 2005), but when their density is decreased via genetic manipulation by $>50 \%$, their dendritic fields are shown to double in areal extent (Poché et al., 2008). Bipolar cells in the primate retina show an increase in dendritic field area as a function of declining cell density at further retinal eccentricities (Telkes et al., 2008), often assumed to reflect a homotypic regulation of dendritic outgrowth via contact inhibition. That variation, however, may arise secondarily, as a function of the differential expansion of more peripheral retina driving passive (interstitial) dendritic elongation (Hitchcock and Easter, 1986; Wong and Collin, 1989; Rodieck and Marshak, 1992). The present study demonstrates directly that modulating the local 

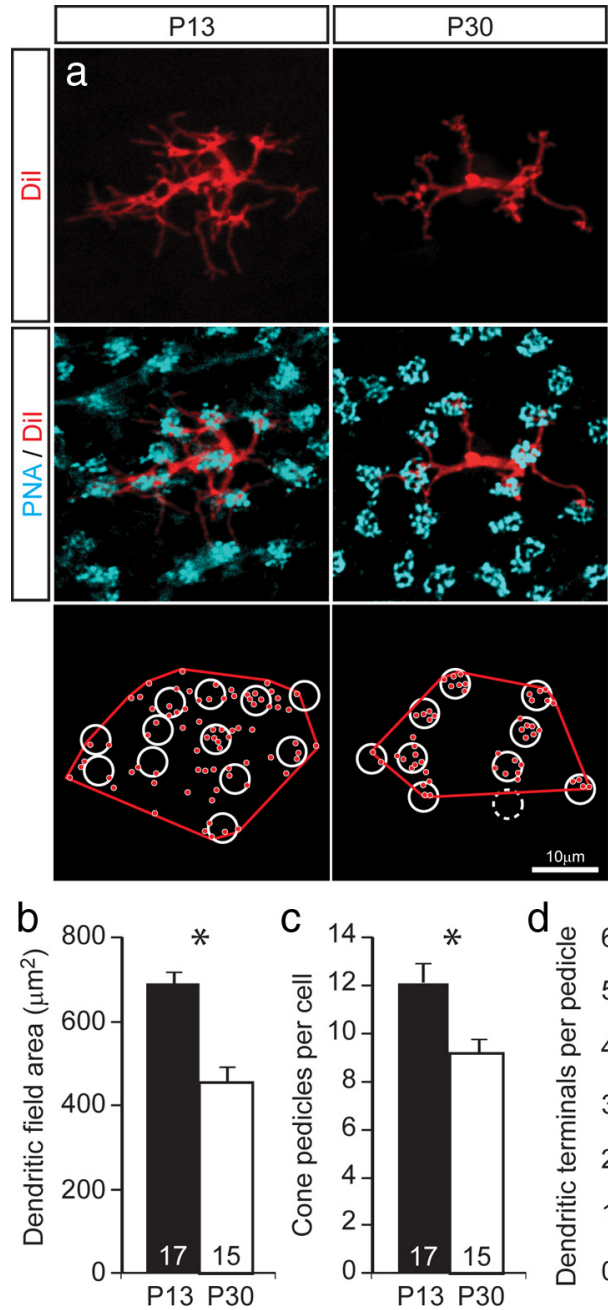
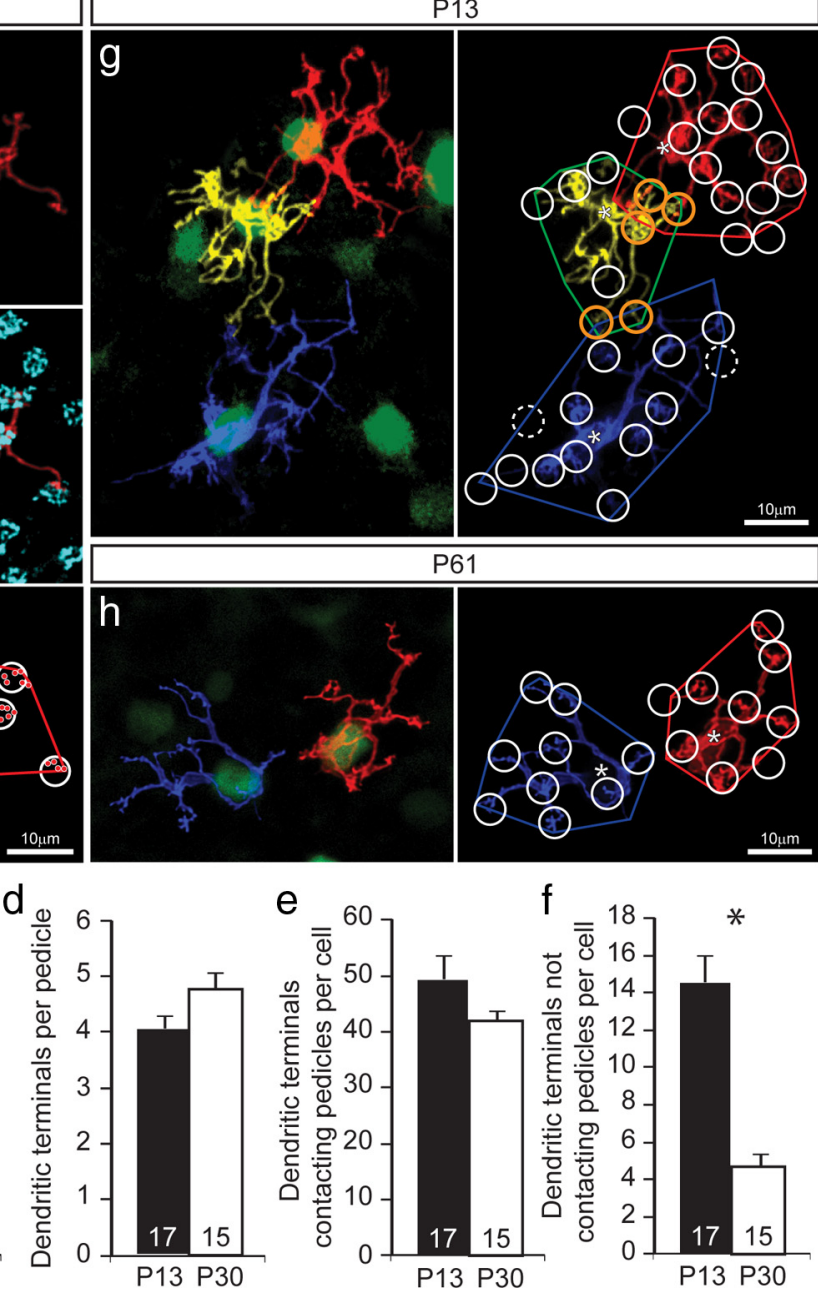

Figure 5. Developing dendritic arbors extend indiscriminately and invade neighboring homotypic territories. $\boldsymbol{a}$, Type 7 bipolar cells form larger dendritic fields with nonselective endings earlier in development. Top panels show individual examples of a Type 7 cone bipolar cell at P13 and P30, in red. Middle panels show the distribution of cone pedicles (blue) relative to the dendritic arbor of these labeled cells. Bottom panels show convex (red) polygons enclosing the dendritic fields, the position of each cone pedicle (white circles), and the locations of each dendritic ending relative to the pedicles. $\boldsymbol{b}, \boldsymbol{c}, \mathrm{P} 13$ cells have significantly larger dendritic field areas than cells at P30 $(p<0.00001)$, and contact significantly more cone pedicles $(p<0.01)$. $\boldsymbol{d}, \boldsymbol{e}$, There is no significant difference in the number of dendritic terminals per cone pedicle, nor the number of dendritic terminals in contact with cone pedicles per cell. $f$, There is, however, an excessive number of dendritic terminals free from pedicles though distributed at this same depth within the OPL $(p<0.00001) . \boldsymbol{g}, \boldsymbol{h}$, Neighboring cells were carefully reconstructed from $z$-stacks of images and pseudocolored for display. P13 cells show dendritic overlap and sharing of cone pedicles (indicated by orange circles; uninnervated pedicles indicated by broken white circles), relative to neighboring mature cells showing exclusive dendritic fields that do not share pedicles. Each asterisk indicates the somal position of the labeled cells, in the right panels; GFP-positive somata, including those labeled with Dil, are shown in the left panels.

frequency of homotypic neighbors, increasing or decreasing them, yields a correspondingly smaller or larger dendritic field area, respectively. This demonstration, the first of its kind in mammalian retina, confirms the homotypic dependency of dendritic field size so commonly assumed within the field.

\section{Homotypic regulation of cone convergence}

Varying the size of the dendritic field in the presence of an invariant population of cone photoreceptors should permit a tiled population of dendritic fields to individually sample a smaller or larger number of cone pedicles, as has been shown previously for horizontal cells (Poché et al., 2008). For the Type 7 cone bipolar cell, both types of mouse cone photoreceptors (M vs UV) are contacted (Breuninger et al., 2011). The fact that the Type 7 cone bipolar cells do indeed now sample fewer or greater numbers of cone pedicles would indicate that this aspect of the dendritic morphology must be independent of any cell-intrinsic specification. Whether there is an upper limit to the size a Type 7 cone bipolar cell dendritic field can achieve, and the number of cone pedicles it can colonize, is unclear; we cannot directly compare the magnitude of the change in dendritic field area to that of the depletion of Type 7 cells because the depletion is not uniform across the retina, and we were undoubtedly biased in seeking regions containing remaining GFP-positive Type 7 cells for DiI injection. Nevertheless, we conclude that the reduction in the density of multiple types of cone bipolar cell has been too severe for each to compensatorily expand its dendritic territory to maintain a coverage of 1.0.

In the present retinas, cone photoreceptor density does not vary with eccentricity, as it does in the primate retina, but the implication of the present findings is that the modulation of cone photoreceptor number in the presence of an unvarying population of bipolar cells should yield bipolar cells of comparable dendritic field area yet showing a direct modulation of their convergence ratios. One-half of this expectation has been borne out, in a study examining the dendritic morphology of Type 7 

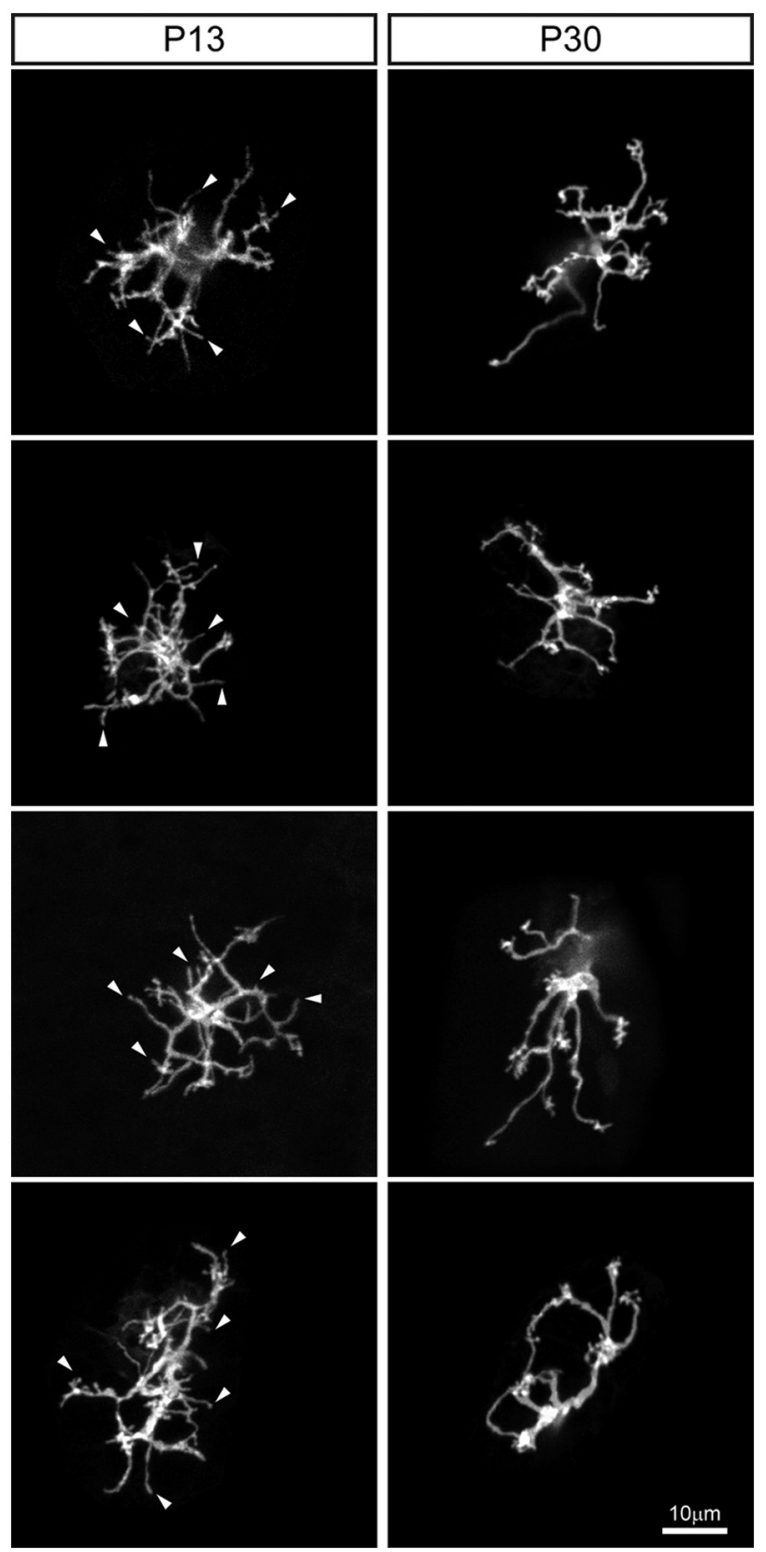

Figure 6. Type 7 cone bipolar cells during development have excessively branched dendritic arbors. Examples of Type 7 cone bipolar cells in the P13 versus P30 wild-type retina, showing the numerous presumptive exploratory endings, confined to the OPL, at the earlier developmental time-point (a few of which are indicated by arrowheads). P30 cells have largely achieved their normal adult morphology by this stage.

cone bipolar cells in the coneless versus conefull mouse retinas (Keeley and Reese, 2010). There, dendritic field area was not affected. Dendritic endings associated with the presence of cones, however, were more difficult to address, as both models showed a sparse and unclustered distribution of dendritic endings that mirrored the lack of large, discrete, PNA-positive synaptic sites in the OPL of these retinas (Keeley and Reese, 2010). Different strains of mice, however, do show a large (e.g., 70\%) variation in cone photoreceptor number (Whitney et al., 2011b), so a direct comparison of Type 7 cone bipolar cells therein, assuming comparable numbers of this population, might permit a direct assessment of this relationship.

\section{Conservation of dendritic terminal number at each pedicle}

The number of dendritic terminals at a pedicle did not show any modulation in the present mouse models, thereby translating into a net decrease or increase in the total number of dendritic endings per Type 7 cone bipolar cell in the Bax KO and Isll CKO retinas, respectively, indicating that total terminal number is also not cell-intrinsically specified. This was somewhat surprising, particularly in the Isl1 CKO retina. Although other bipolar cell types were also decreased (Elshatory et al., 2007), we were of course only assessing those dendritic terminals belonging to Type 7 cone bipolar cells that managed to expand their dendritic arbors and colonize more pedicles, yet at each of these pedicles, the number of dendritic endings was not significantly different. This suggests that heterotypic interactions with other postsynaptic cells do not limit the number of endings a Type 7 cone bipolar cell makes at a cone pedicle; rather, the cone pedicle appears to constrain this feature. Interestingly, the number of horizontal cell endings at a pedicle is also constrained, but the number contributed by each of the six overlapping horizontal cell dendritic fields changes through development, favoring the horizontal cells closer to a given pedicle at the expense of those farther removed from it (Reese et al., 2005).

\section{Dendritic overlap precedes tiling}

The outgrowing dendrites of Type 7 cone bipolar cells extend multiple, presumably exploratory, endings as they investigate their local environment. The present study has shown that these processes localize to the innermost stratum of the OPL, where cone pedicles are positioned, yet they do not target the cone pedicles as precisely as they will at later developmental stages. Whether these immature Type 7 cone bipolar cells contact rod spherules is unknown, but since this cell type exhibits a few contacts free of pedicles in maturity (Keeley and Reese, 2010; present results), when they receive limited rod input directly (Tsukamoto et al., 2007), these endings are certainly situated among an alternative potential presynaptic partner. During this phase of dendritic outgrowth, these dendrites also do not respect the dendritic boundaries defined by their homotypic neighbors, as they largely do at later developmental stages. In the adult retina, while occasional pedicles at the boundary are shared by neighboring Type 7 cone bipolar cells (Wässle et al., 2009), they more commonly apportion the territory exclusively. Note that the two neighboring somata in Figure $5 \mathrm{~h}$ are positioned relatively close to one another (evidenced by the asterisks), yet they avoid sharing contacts with any pedicles along this shared boundary. Developing bipolar cells, by contrast, produce larger dendritic fields with endings localized to significantly more pedicles than at later stages, suggesting greater coinnervation via overlapping dendrites by homotypic neighbors. This was in fact borne out by labeling neighboring Type 7 cells at this early developmental stage (Fig. $5 g$ ). These results make clear that the dendritic tiling observed in maturity is not a simple consequence of contact inhibition at the time of dendritic outgrowth. Rather, dendritic fields initially overlap, subsequently retracting until they achieve a coverage of 1.0. This retraction should therefore be greater in the Bax KO retina, and less in the Isl1 CKO retina, but we cannot rule out the possibility that the latter condition may include some cells that actively increase in size due to a loss of all immediate neighbors.

Homotypic interactions apparently drive this retraction to minimize dendritic overlap during the very period of pedicle colonization. The role played by homotypic neighbors cannot be an indirect one, through the pedicles themselves, since the dendritic field size of Type 7 cells is not altered in coneless mutant mice (Keeley and Reese, 2010). Rather, both the size of the dendritic field and the connectivity of this cell with its afferent pop- 
ulation depend upon a direct interaction between homotypic neighbors. Circuitry at the first synapse in the retina is therefore shown to be conspicuously plastic and dependent upon like-type neighboring cells within the local environment.

\section{References}

Bleckert A, Wong ROL (2011) Identifying roles for neurotransmission in circuit assembly: insights gained from multiple model systems and experimental approaches. Bioessays 33:61-72.

Breuninger T, Puller C, Haverkamp S, Euler T (2011) Chromatic bipolar cell pathways in the mouse retina. J Neurosci 31:6504-6517.

Chang B, Dacey MS, Hawes NL, Hitchcock PF, Milam AH, Atmaca-Sonmez P, Nusinowitz S, Heckenlively JR (2006) Cone photoreceptor function loss-3, a novel mouse model of achromatopsia due to a mutation in Gnat2. Invest Ophthalmol Vis Sci 47:5017-5021.

Elshatory Y, Everhart D, Deng M, Xie X, Barlow RB, Gan L (2007b) Islet-1 controls the differentiation of retinal bipolar and cholinergic amacrine cells. J Neurosci 27:12707-12720.

Espinosa JS, Wheeler DG, Tsien RW, Luo L (2009) Uncoupling dendrite growth and patterning: single-cell knockout analysis of NMDA receptor 2B. Neuron 62:205-217.

Haverkamp S, Grünert U, Wässle H (2000) The cone pedicle, a complex synapse in the retina. Neuron 27:85-95.

Hitchcock PF, Easter SS Jr (1986) Retinal ganglion cells in goldfish: a qualitative classification into four morphological types, and a quantitative study of the development of one of them. J Neurosci 6:1037-1050.

Holtmaat A, Svoboda K (2009) Experience-dependent structural synaptic plasticity in the mammalian brain. Nat Rev Neurosci 10:647-658.

Huang L, Max M, Margolskee RF, Su H, Masland RH, Euler T (2003) The G protein subunit Gg13 is co-expressed with Gao and Gb3 in retinal On bipolar cells. J Comp Neurol 455:1-10.

Jan YN, Jan LY (2010) Branching out: mechanisms of dendritic arborization. Nat Rev Neurosci 11:316-328.

Keeley PW, Reese BE (2010) Role of afferents in the differentiation of bipolar cells in the mouse retina. J Neurosci 30:1677-1685.

Kerschensteiner D, Morgan JL, Parker ED, Lewis RM, Wong ROL (2009) Neurotransmission selectively regulates synapse formation in parallel circuits in vivo. Nature 460:1016-1020.

Li J, Erisir A, Cline H (2011) In vivo time-lapse imaging and serial section electron microscopy reveal developmental synaptic rearrangements. Neuron 69:273-286.

Lin B, Wang SW, Masland RH (2004) Retinal ganglion cell type, size, and spacing can be specified independent of homotypic dendritic contacts. Neuron 43:475-485.

Morgan JL, Dhingra A, Vardi N, Wong RO (2006) Axons and dendrites originate from neuroepithelial-like processes of retinal bipolar cells. Nat Neurosci 9:85-92.

Ogilvie JM, Deckwerth TL, Knudson CM, Korsmeyer SJ (1998) Suppression of developmental retinal cell death but not photoreceptor degeneration in Bax-deficient mice. Invest Ophthalmol Vis Sci 39:1713-1720.

Olney JW (1968) An electron microscopic study of synapse formation, receptor outer segment development, and other aspects of developing mouse retina. Invest Ophthalmol 7:250-268.

Péquignot MO, Provost AC, Sallé S, Taupin P, Sainton KM, Marchant D, Martinou JC, Ameisen JC, Jais J-P, Abitbol M (2003) Major role of BAX in apoptosis during retinal development and in establishment of a functional postnatal retina. Dev Dyn 228:231-238.

Poché RA, Raven MA, Kwan KM, Furuta Y, Behringer RR, Reese BE (2008) Somal positioning and dendritic growth of horizontal cells are regulated by interactions with homotypic neighbors. Eur J Neurosci 27:1607-1614.

Reese BE, Raven MA, Stagg SB (2005) Afferents and homotypic neighbors regulate horizontal cell morphology, connectivity and retinal coverage. J Neurosci 25:2167-2175.

Rodieck RW, Marshak DW (1992) Spatial density and distribution of choline acetyltransferase immunoreactive cells in human, macaque, and baboon retinas. J Comp Neurol 321:46-64.

Rowan S, Cepko CL (2004) Genetic analysis of the homeodomain transcription factor Chx10 in the retina using a novel multifunctional BAC transgenic mouse reporter. Dev Biol 271:388-402.

Sherry DM, Wang MM, Bates J, Frishman LJ (2003) Expression of vesicular glutamate transporter 1 in the mouse retina reveals temporal ordering in development of rod vs. cone and ON vs. OFF circuits. J Comp Neurol 465:480-498.

Sun Y, Liang X, Najafi N, Cass M, Lin L, Cai CL, Chen J, Evans SM (2007) Islet 1 is expressed in distinct cardiovascular lineages, including pacemaker and coronary vascular cells. Dev Biol 304:286-296.

Telkes I, Lee SC, Jusuf PR, Grünert U (2008) The midget-parvocellular pathway of marmoset retina: a quantitative light microscopic study. J Comp Neurol 510:539-549.

Tsukamoto Y, Morigiwa K, Ishii M, Takao M, Iwatsuki K, Nakanishi S, Fukuda Y (2007) A novel connection between rods and ON cone bipolar cells revealed by ectopic metabotropic glutamate receptor 7 (mGluR7) in mGluR6-deficient mouse retinas. J Neurosci 27:6261-6267.

Wässle H, Puller C, Müller F, Haverkamp S (2009) Cone contacts, mosaics and territories of bipolar cells in the mouse retina. J Neurosci 29:106-117.

Whitney IE, Keeley PW, Raven MA, Reese BE (2008) Spatial patterning of cholinergic amacrine cells in the mouse retina. J Comp Neurol 508:1-12.

Whitney IE, Raven MA, Ciobanu DC, Poché RA, Ding Q, Elshatory Y, Gan L, Williams RW, Reese BE (2011a) Genetic modulation of horizontal cell number in the mouse retina. Proc Natl Acad Sci U S A 108:9697-9702.

Whitney IE, Raven MA, Lu L, Williams RW, Reese BE (2011b) A QTL on chromosome 10 modulates cone photoreceptor number in the mouse retina. Invest Ophthalmol Vis Sci 52:3228-3236.

Wong ROL, Collin SP (1989) Dendritic maturation of displaced putative cholinergic amacrine cells in the rabbit retina. J Comp Neurol 287:164178. 